\title{
Distribution spatio-temporelle du zooplancton en relation avec les facteurs abiotiques dans un hydrosystème urbain : le ruisseau Kondi (Douala, Cameroun)
}

\author{
ONANA Fils Mamert1,2, ZEBAZE TOGOUET Serge Hubert ${ }^{*}$, NYAMSI TCHATCHO Nectaire Lié1,2, \\ DOMCHE TEHAM Henry Bruno ${ }^{1}$, NGASSAM Pierre ${ }^{1}$ \\ 1 Unité d'Hydrobiologie et Environnement, Laboratoire de Biologie Générale, Faculté des Sciences, Université de \\ Yaoundé I, P.O. Box 812, Yaoundé, Cameroun. \\ 2 Laboratoire d'Aquaculture et Démographie des Ressources Halieutiques, Institut des Sciences \\ Halieutiques, Université de Douala, P.O. Box 7236, Douala, Cameroun. \\ *Corresponding author email: zebasehu@yahoo.fr Tel: 0023777453020 / 99026839
}

Original submitted in on 1st July 2014. Published online at www.m.elewa.org on 31st October 2014. http://dx.doi.org/10.4314/jab.v82i1.6

Objectif : Une étude visant à déterminer la distribution spatio-temporelle du zooplancton en relation avec les variables environnementales du ruisseau Kondi, cours d'eau du réseau hydrographique du Wouri qui arrose Douala, ville portuaire et métropole économique du Cameroun a été menée d'avril 2011 à mai 2012.

Méthodologie et résultats : Trois sites, situés respectivement dans les cours supérieur, moyen et inférieur ont été échantillonnés. Les teneurs élevées des variables physicochimiques étudiées montrent que les eaux du Kondi sont eutrophes à hypereutrophes. Le zooplancton constitué de 56 espèces dont 40 rotifères, 12 cladocères, 3 copépodes et 01 gastrotriche est dominé par les rotifères qui représentent $75,90 \%$ de l'abondance totale. L'espèce Rotaria rotaria est la plus abondante avec une abondance relative de $36,73 \%$. La communauté zooplanctonique présente les effectifs les plus élevés pendant la saison sèche (décembre, janvier, février) lorsque les concentrations en nutriments sont les plus fortes dans le cours d'eau.

Conclusion et applications : Le coefficient de corrélation de Spearmann a permis de révéler des liaisons significatives entre Rotaria rotaria, Cephalodella gibba, Platyias quadricornis, Rotaria neptunia, Mytilina bisulcata, espèces caractéristiques des milieux eutrophes et les fortes valeurs de quelques variables environnementales telles que la conductivité électrique, les orthophosphates et l'azote ammoniacal, confirmant ainsi la grande dégradation de ces eaux et l'usage de ces espèces comme indicatrices de la pollution en milieu lotique.

Mots clés : zooplancton, eutrophisation, distribution spatio-temporelle, rivière Kondi, Douala 


\title{
Onana et al. J. Appl. Biosci. 2014. Distribution spatio-temporelle du zooplancton en relation avec les facteurs abiotiques dans un hydrosystème urbain : le ruisseau Kondi, Cameroun.
}

Spatio-temporal distribution of zooplankton in relation with the abiotics factors in an urban hydrosystem : the Kondi stream (Douala, Cameroon)

\begin{abstract}
Objectives: A study aimed at determining the spatiotemporal distribution of zooplankton in relation with the environmental variables of the Kondi, a watercourse of Wouri hydrographic basin, harbour city and economic metropolis of Cameroon was led from April 2011 to May 2012.

Methodology and Results: Three sites, situated respectively in the upper, mid and lower watercourses were sampled. The high values variables studied showed that waters of the Kondi are eutrophic to hypereutrophic. The zooplankton constituted of 56 species of which 40 rotifers, 12 cladocerans, 3 copepods and 1 gastrotriche which was dominated by the rotifers which represented $75.90 \%$ of the total abundance of the zooplankton. This fauna was dominated by the species $R$. rotaria which constituted $36.73 \%$ of the zooplankton community. These zooplanctonic communities reached its maximum development during the dry season (December, January, February) when nutrient concentrations were highest in the watercourse.

Conclusion and application: The Spearmann's coefficient of correlation revealed relations between $R$. rotaria, C. gibba, P. quadricornis, R. neptunia and Mytilina bisulcata, species indicators of eutrophic hydrosystems and the high values of some environmental variables such as electric conductivity, orthophosphates and ammonia nitrogen, confirms the strongly polluted character of these waters and the potential used of zooplankton communities as indicator of water quality even in lotic milieu.
\end{abstract}

Keywords: zooplankton, eutrophication, spatio-temporal distribution, Kondi stream, Douala.

\section{INTRODUCTION}

Les cours d'eau procurent une variété de biens et de services aux sociétés humaines (Costanza et al., 1997). Pourtant, dans la majorité des pays en voie de développement, la plupart des cours d'eau urbains sont utilisés pour l'évacuation des déchets des villes et des industries (Sikati Foko, 1998). II s'en suit une dégradation de la qualité de l'eau, une modification des peuplements et le plus souvent, une diminution de la biodiversité (Zébazé Togouet, 2008). Malgré leur importance et la sévérité des menaces auxquelles ils font face, les écosystèmes d'eau courante ne bénéficient que d'une très faible part des évaluations environnementales et du suivi à l'échelle de la planète (Amis et al., 2007). On comprend dès lors l'urgence de l'étude de ces écosystèmes et de la maîtrise de leur fonctionnement pour une gestion durable de la ressource (Foto Menbohan et al., 2011a). Etablir un lien de causalité entre les perturbations dans l'environnement et divers paramètres biotiques est fondamental afin de comprendre l'impact réel des activités anthropiques sur les communautés écologiques des cours d'eau (Novotny et al., 2005). Le zooplancton, abondant dans les eaux douces et très sensibles aux variations des conditions de son milieu, pourrait être utilisé pour établir un tel lien et servir par conséquent d'outils d'évaluation de l'état de santé des systèmes lotiques (Sladecek, 1983 ; Nogrady et al., 1993 ; Moss, 1998 ; Zébazé Togouet, 2000). Au Cameroun, très peu d'études ont été consacrées au zooplancton des hydrosystèmes lotiques. En effet, seuls les travaux de Foto Menbohan et al. (2006) et de Foto Menbohan et al. (2011b) mentionnent ces organismes dans les eaux courantes du Cameroun. Ces travaux ont révélé que les cours d'eau de Yaoundé sont sujets à une pollution organique d'origine anthropique. La présente étude vise à décrire les variations spatiotemporelles des abondances du zooplancton en relation avec quelques variables abiotiques du Kondi, un cours d'eau de la ville de Douala, ville industrielle caractérisée par une quasi absence d'unités de traitement des eaux usées (Kramkimel et al., 2004). 


\section{Onana et al. J. Appl. Biosci. 2014. Distribution spatio-temporelle du zooplancton en relation avec les facteurs abiotiques dans un hydrosystème urbain : le ruisseau Kondi, Cameroun.}

\section{MATERIEL ET METHODES}

Description de la zone d'étude: Douala, capitale économique du Cameroun et chef-lieu de la région du Littoral, est une agglomération qui s'étale sur près de 18 000 hectares pour une population estimée à 2755011 habitants (BUCREP, 2010). Elle s'étend entre 04 $03^{\circ}$ et $04^{\circ} 57^{\prime}$ de latitude Nord et entre $09^{\circ} 42^{\prime}$ et $09^{\circ} 47^{\prime}$ de longitude Est. D'altitude moyenne $13 \mathrm{~m}$, Douala est soumise au climat équatorial de type guinéen et de sous type camerounéen, caractérisé par deux saisons : Une saison pluvieuse de neuf mois (mars - novembre) et une courte saison sèche, de décembre à février (Suchel, 1972). Les précipitations y sont abondantes et régulières avec des valeurs annuelles variant entre $2596 \mathrm{~mm}$ et 5 $328 \mathrm{~mm}$. La température de l'air dans la ville de Douala est relativement élevée avec une moyenne mensuelle d'environ $28^{\circ} \mathrm{C}$ (Suchel 1972). D'une superficie d'environ $10,375 \mathrm{~km}^{2}$, le bassin versant du Kondi est situé entre $04^{\circ} 01^{\prime} 87^{\prime \prime}$ et $04^{\circ} 04^{\prime} 18^{\prime \prime}$ de latitude Nord et entre $09^{\circ} 45^{\prime} 78^{\prime \prime}$ et $09^{\circ} 46^{\prime} 89^{\prime \prime}$ de longitude Est. D'une longueur d'environ $5,9 \mathrm{~km}$, le cours d'eau Kondi prend source au quartier Ndog-Mbè, et arrose les quartiers Songbikako, Logbaba, Cité des palmiers, Beedi et Ndog-Bong. II traverse quelques zones marécageuses dont la plus importante est celle allant du carrefour Cité des Palmiers à l'Hôpital Général de Douala au quartier Beedi. Le ruisseau Kondi sert parfois de dépotoir aux populations riveraines et reçoit ainsi divers altéragènes organique et inorganique qui sont susceptibles de modifier la qualité de ses eaux (Priso et al., 2012). Ce cours d'eau qui ne bénéficie d'aucun suivi de la part des municipalités et dont les eaux sont quotidiennement utilisées par les populations riveraines devrait pourtant faire l'objet d'un suivi régulier. Dans le cadre de cette étude trois stations ont été choisies. Les deux premières stations K1 et K2 encadrent la portion du Kondi recevant les effluents de la zone industrielle de Ndokoti. Ces stations permettront d'apprécier l'effet de quelques effluents industriels, principalement de la Société Anonyme des Brasseries du Cameroun (SABC) sur la physico-chimie et le zooplancton du Kondi. La station K3 quant à elle, est située dans une zone marécageuse riche en Echinocloa pyramidalis et Paspalum conjugatum, macrophytes souvent utilisés dans les stations de phytoépuration des eaux usées à Yaoundé. Cette station a permis d'apprécier l'effet d'un marécage sur la biodiversité aquatique et sur la qualité de l'eau. Par ailleurs, il fallait que les trois parties du cours d'eau soient représentés : - La station K1 (0402'16,9"N et 0946'20,5"E), d'altitude $16 \mathrm{~m}$, est localisée dans le cours supérieur du Kondi au quatier Ndog-Mbè, à environ $250 \mathrm{~m}$ de la source, dans un environnement marécageux très peu peuplée;

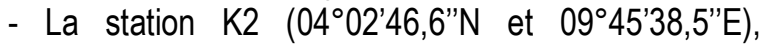
d'altitude $15 \mathrm{~m}$, est située dans le cours moyen du Kondi, au lieu dit carrefour Cité des Palmiers, à environ 2,4 Km de la source. A quelques dizaines de mètres en amont de cette station, viennent se déverser dans le Kondi, les effluents de la SABC, de la Cotonnière Industrielle du Cameroun (CICAM) et de divers marchés situés au quartier Ndokoti ;

- La station K3 $\left(04^{\circ} 04^{\prime} 00,4^{\prime \prime} \mathrm{N}\right.$ et 945'13,3'E), d'altitude $11 \mathrm{~m}$, est située dans le cours inférieur, au quartier NdogBong, en contrebas du collège "La Conquête", dans une zone marécageuse et approximativement à $5,3 \mathrm{Km}$ de la source.

Techniques de prélèvement et d'analyse des échantillons : Les échantillons d'eau destinés à l'analyse physico-chimique ont été prélevés dans les trois stations en matinée, entre 8 heures et 10 heures, d'avril 2011 à mai 2012, suivant une fréquence mensuelle. Ces prélèvements d'eau ont été faits à contre courant, en prenant soin de ne pas faire de bulles, dans des flacons à double bouchage en polyéthylène de $1000 \mathrm{~mL}$, puis transportés au laboratoire dans une enceinte réfrigérée. Pour le zooplancton, $10 \mathrm{~L}$ d'eau prélevée à l'aide d'un seau en polyéthylène de contenance $10 \mathrm{~L}$, au niveau des faciès lentiques et après touillage de l'herbier ont été passés à travers un filet à plancton de $64 \mu \mathrm{m}$ d'ouverture de maille. Le processus est répété dix fois à chaque station, jusqu'à filtration d'un volume total de $100 \mathrm{~L}$ d'eau. Ensuite le filet est rincé à l'eau de l'échantillon dans le sens contraire de celui de la filtration pour ne perdre aucun organisme ; l'échantillon de zooplancton obtenu $(200 \mathrm{~mL})$ est introduit dans deux flacons de $150 \mathrm{~mL}$ chacun. Le premier flacon contient une moitié de l'échantillon qui est fixée sur le terrain avec du formol 5 $\%$ et servira au dénombrement des organismes loriqués. La seconde moitié (non fixée) contenue dans le deuxième flacon sera utilisée pour les dénombrements des espèces qui se déforment sous l'action du formol. Au laboratoire, les organismes zooplanctoniques ont été identifiés à l'aide du microscope Olympus CK2 UL WCD 0.30 en utilisant les clés et ouvrages de Koste (1978), Durand et Lévêque (1980), Pourriot et Francez (1986), Shiel (1995), Segers (1996), Zébazé Togouet (2000) et Fernando (2002) suivant les techniques classiques. Les dénombrements ont ensuite été effectués à la loupe binoculaire de marque WILD M5 en triplicat. En effet, 10 $\mathrm{mL}$ d'échantillon homogénéisé ont été prélevés à l'aide d'une pipette étalonnée automatique et introduits dans 


\section{Onana et al. J. Appl. Biosci. 2014. Distribution spatio-temporelle du zooplancton en relation avec les facteurs abiotiques dans un hydrosystème urbain : le ruisseau Kondi, Cameroun.}

une boîte de Pétri quadrillée de $30 \mathrm{~mm}$ de diamètre dans laquelle les dénombrements ont été effectués sur trois prises de $10 \mathrm{~mL}$ chaque fois. La densité des individus a été calculée en utilisant la formule : $D=(n \times v) / V$; où $D$ est la densité (exprimée en individus par litre), v est le volume total de l'échantillon analysé $(v=200 \mathrm{~mL})$ et $V$ est le volume d'eau filtrée sur le terrain $(\mathrm{V}=100 \mathrm{~L})$.

Analyses physico-chimiques : La température et la conductivité électrique ont été mesurées in situ, respectivement à l'aide d'un thermomètre à colonne de mercure gradué au $1 / 10^{\circ} \mathrm{C}$ et d'un conductimètre de marque Wagtech. Le $\mathrm{pH}$ et les teneurs en oxygène dissous ont également été mesurés in situ à l'aide d'un multimètre de marque $\mathrm{HACH} \mathrm{HQ14d.} \mathrm{Au} \mathrm{laboratoire,} \mathrm{la}$ turbidité de l'eau, les teneurs de l'eau en Matières En Suspension (MES), en orthophosphates et quelques formes d'azote (azote ammoniacal et nitrite) ont été évaluées au spectrophotomètre HACHDR/2800 selon les méthodes de APHA (1998). La détermination de l'oxydabilité s'est faite par volumétrie suivant la norme AFNOR NFT 90-050 au moyen du permanganate de potassium après décoloration au sel de Mohr.

Analyses des données: Le traitement des données physico-chimiques est basé sur le calcul de l'indice de pollution organique (IPO) (Leclercq et Maquet, 1987). Cet indice s'obtient au moyen des valeurs de la $\mathrm{DBO}_{5}$, nitrites, phosphates et ammonium. Dans la présente étude, la $\mathrm{DBO}_{5}$ n'ayant pu être mesurée, l'indice a été calculé à partir des trois variables : azote ammoniacal $\left(\mathrm{NH}_{4}{ }^{+}\right)$, nitrites $\left(\mathrm{NO}_{2}{ }^{-}\right)$, et orthophosphates $\left(\mathrm{PO}_{4}{ }^{3-}\right)$. Leclercq (2001) indique que l'on peut obtenir cet indice avec les valeurs de ces trois paramètres, le principe du calcul étant de répartir les valeurs des trois éléments polluants (azote ammoniacal, nitrites orthophosphates) en cinq classes et de déterminer à partir des valeurs obtenues dans l'étude, le numéro de classe correspondant pour chaque paramètre en se servant des données moyennes du Tableau 1.

Tableau 1 : Limites des classes de l'Indice de Pollution Organique (Leclercq, 2001)

\begin{tabular}{llll}
\hline Classes & $\mathrm{NH}_{4}{ }^{+}(\mathrm{mg} / \mathrm{L})$ & $\mathrm{NO}_{2}^{-}(\mu \mathrm{g} / \mathrm{L})$ & $\mathrm{PO}_{4}^{3-}(\mu \mathrm{g} / \mathrm{L})$ \\
\hline 5 & $<0,1$ & $<5$ & $<15$ \\
4 & $0,1-0,9$ & $6-10$ & $16-75$ \\
3 & $1-2,4$ & $11-50$ & $76-250$ \\
2 & $2,5-6$ & $51-150$ & $251-900$ \\
1 & $>6$ & $>150$ & $>900$ \\
\hline
\end{tabular}

$\mathrm{IPO}=$ moyenne des numéros des classes des 03 paramètres:

IPO $=1,0-1,9:$ pollution organique très forte.

$\mathrm{IPO}=2,0-2,9:$ pollution organique forte.

IPO $=3,0-3,9:$ pollution organique modérée.

IPO $=4,0-4,5:$ pollution organique faible.

IPO = 4,6-5,0: pollution organique nulle.

L'analyse des données biologiques quant à elle, est basée sur le calcul des indices de diversité et des corrélations.

- La fréquence d'occurrence $(F)$ d'une espèce est le rapport du nombre de prélèvements ou cette espèce est notée au nombre total de prélèvements effectués.

$\mathrm{F}=\left(\mathrm{P}_{\mathrm{a}} / \mathrm{P}\right) \times 100$.

Avec: $\mathrm{Pa}=$ nombre total de prélèvements contenant l'espèce prise en considération. $P$ est le nombre total de prélèvements faits. Selon Dajoz (1982), on distingue : les espèces constantes ( $F \geq 50 \%$ ), les espèces accessoires $(25 \%<F<50 \%)$ et les espèces accidentelles ( $F \leq 25$ $\%)$.

- L'indice de diversité de Shannon et Weaver (1949), a permis de caractériser la structure des peuplements. Sa formule est $H^{\prime}=-\sum_{i=1}^{n} P_{i} \log _{2} P_{i}$ où, $\mathrm{P}_{\mathrm{i}}=$ abondance relative du taxon i.

- L'équitabilité $(\mathrm{J})$ de Piélou a permis de mesurer la répartition des espèces du peuplement par rapport à une répartition théorique égale pour l'ensemble des espèces (Barbault, 1995).

$\mathrm{J}=\frac{\mathrm{H} r}{\log _{2} \mathrm{~S}}$ avec $: \mathrm{H}^{\prime}=$ indice de Shannon et Weaver et

$S=$ nombre total de taxons dans l'échantillon. Les valeurs de l'équitabilité varie de 0 (dominance d'une des espèces) à 1 (équirépartition des individus dans les différentes espèces).

- La corrélation de rang de Spearmann a permis d'établir le degré de liaison entre variables physico-chimiques et variables biologiques pour mieux caractériser l'état du milieu. Cette corrélation a été calculé à l'aide du logiciel SPSS 15.0 et a été considérée comme significatives pour $a=0,05$. 


\section{RESULTATS}

Caractéristiques physico-chimiques : Les résultats des analyses physico-chimiques des eaux du Kondi sont réunis dans le tableau 2. La température des eaux du Kondi a varié de $23,5^{\circ} \mathrm{C}$ à $32,4^{\circ} \mathrm{C}$. Globalement, les températures les plus faibles sont enregistrées dans le cours inférieur (K3) dont la température moyenne est de $26,59^{\circ} \mathrm{C}$ alors que les plus fortes températures sont obtenues dans le cours supérieur (K1) qui a une température moyenne de $27,04^{\circ} \mathrm{C}$. Les valeurs les plus fortes des MES et de la turbidité sont observées à la station K2 à tous les mois sauf aux mois de septembre et octobre 2011 alors que les plus faibles sont notées à la station K1. Les valeurs de la conductivité électrique varient de $56 \mu \mathrm{S} / \mathrm{cm}$ (K1, juillet 2011) à $978 \mu \mathrm{S} / \mathrm{cm}(\mathrm{K} 2$, mai 2011). En outre, la moyenne au niveau de chaque point d'échantillonnage montre que la conductivité électrique croît de l'amont vers l'aval du Kondi. Le pH varie de 5,42 et 10,18, avec les plus fortes valeurs à la station K2. Les teneurs en azote ammoniacal et en orthophosphates fluctuent respectivement de 0,00 à 19,2 $\mathrm{mg} / \mathrm{L} \mathrm{NH}_{4}{ }^{+}$et de 0,00 à $5,18 \mathrm{mg} / \mathrm{L}$ de $\mathrm{PO}_{4}{ }^{3-}$.
Globalement, ces teneurs augmentent progressivement de la station $\mathrm{K} 1$ à la station $\mathrm{K} 2$ et diminuent de la station K2 à la station K3. Les concentrations en nitrites sont restées très faibles tout au long de l'étude. Elles sont très souvent en dessous de $0,3 \mathrm{mg} / \mathrm{L}$, sauf au mois de juillet 2011 et avril 2012 respectivement aux stations K2 et K1. La teneur de l'eau en oxygène dissous a varié entre $3 \%$ et $115 \%$. La station K1 présente la saturation moyenne la plus élevées $(69,36 \pm 28,79 \%$ ) alors que la station K2 montre la saturation moyenne la plus faible $(32,61 \pm$ $34,99 \%$ ). Pour ce qui est de l'oxydabilité, la station K1 a enregistré les valeurs les plus faibles avec une moyenne de 3,08 $\pm 2,17 \mathrm{mg} / \mathrm{L}$ d'oxygène alors que la station $\mathrm{K} 2$ montre les plus fortes teneurs de ce paramètre avec une moyenne de 6,68 $\pm 3,11 \mathrm{mg} / \mathrm{L}$ d'oxygène. L'IPO oscille entre 1,67 (pollution organique forte) obtenu en avril 2012 à toutes les stations et 4 (pollution organique faible) enregistré à la station K3 en Mai 2011. Globalement, les valeurs moyennes de l'IPO à chaque station indiquent une pollution organique forte à toutes les stations.

Tableau 2 : Valeurs moyennes \pm écarts types de quelques paramètres physicochimiques aux différentes stations d'étude du Kondi. (Entre parenthèse valeurs minimales et maximales)

\begin{tabular}{|c|c|c|c|}
\hline Stations d'échantillonnage & K1 & K2 & K3 \\
\hline Température $\left({ }^{\circ} \mathrm{C}\right)$ & $\begin{array}{l}27,04 \pm 1,64 \\
(24,0-31,0)\end{array}$ & $\begin{array}{l}27,02 \pm 2,10 \\
(24,0-32,0)\end{array}$ & $\begin{array}{l}26,59 \pm 2,34 \\
(24,0-32,0)\end{array}$ \\
\hline MES (mg/L) & $\begin{array}{l}10,5 \pm 6,38 \\
(0,0-23,0)\end{array}$ & $\begin{array}{c}37,07 \pm 19,45 \\
(4,0-78,0)\end{array}$ & $\begin{array}{c}15,43 \pm 13,29 \\
(2,0-55,0)\end{array}$ \\
\hline Turbidité (NTU) & $\begin{array}{c}13,75 \pm 14,54 \\
(2,5-57,0)\end{array}$ & $\begin{array}{c}26,57 \pm 23,51 \\
(0,0-79,0)\end{array}$ & $\begin{array}{c}25,29 \pm 21,2 \\
(5,0-81,0)\end{array}$ \\
\hline $\mathrm{pH}$ & $\begin{array}{c}6,52 \pm 0,65 \\
(5,4-7,3)\end{array}$ & $\begin{array}{c}7,25 \pm 0,92 \\
(5,6-9,3)\end{array}$ & $\begin{array}{c}7,17 \pm 1,0 \\
(5,7-10,0)\end{array}$ \\
\hline Conductivité électrique $(\mu \mathrm{S} / \mathrm{cm})$ & $\begin{array}{l}172,5 \pm 126,4 \\
(56,0-587,0)\end{array}$ & $\begin{array}{r}321,7 \pm 218,5 \\
(110,0-978,0)\end{array}$ & $\begin{array}{c}326 \pm 223,1 \\
(109,0-881,0)\end{array}$ \\
\hline Azote ammoniacal $\left(\mathrm{mg} / \mathrm{L} \mathrm{N}-\mathrm{NH}_{4}\right)$ & $\begin{array}{c}1,29 \pm 2,33 \\
(0,1-8,0)\end{array}$ & $\begin{array}{l}2,89 \pm 2,2 \\
(0,1-8,7)\end{array}$ & $\begin{array}{l}3,57 \pm 4,8 \\
(0,0-19,0)\end{array}$ \\
\hline Orthophosphates (mg/L P-PO & $\begin{array}{l}0,8 \pm 1,31 \\
(0,0-4,8)\end{array}$ & $\begin{array}{c}2,42 \pm 1,64 \\
(0,0-4,6)\end{array}$ & $\begin{array}{l}1,1 \pm 1,36 \\
(0,0-5,2)\end{array}$ \\
\hline Nitrites (mg/L N-NO ${ }_{2}$ ) & $\begin{array}{c}0,18 \pm 0,17 \\
(0,0-0,7)\end{array}$ & $\begin{array}{l}0,08 \pm 0,1 \\
(0,0-0,4)\end{array}$ & $\begin{array}{l}0,05 \pm 0,08 \\
(0,0-0,03)\end{array}$ \\
\hline Oxygène dissous (\% sat) & $\begin{array}{l}69,36 \pm 28,79 \\
(16,0-113,0)\end{array}$ & $\begin{array}{c}32,61 \pm 34,99 \\
(3,0-88,0)\end{array}$ & $\begin{array}{c}38,17 \pm 35,36 \\
(4,3-115,0)\end{array}$ \\
\hline Oxydabilité (mg/L d'oxygène) & $\begin{array}{c}3,08 \pm 2,17 \\
(0,0-6,5)\end{array}$ & $\begin{array}{l}6,68 \pm 3,11 \\
(2,9-14,0)\end{array}$ & $\begin{array}{l}4,65 \pm 2,45 \\
(0,9-11,0)\end{array}$ \\
\hline IPO & $\begin{array}{c}2,64 \pm 0,66 \\
(1,67-3,67)\end{array}$ & $\begin{array}{l}2,16 \pm 0,31 \\
(1,67-3,0)\end{array}$ & $\begin{array}{l}2,62 \pm 0,62 \\
(1,67-4,0)\end{array}$ \\
\hline
\end{tabular}


Caractéristiques biologiques

Composition spécifique du peuplement

zooplanctonique : Au cours de cette étude, 56 espèces zooplanctoniques dont 40 de rotifères, 12 de cladocères, 03 de copépodes et 01 espèce de gastrotriche ont été identifiées dans le Kondi. Des nauplii et des copépodites ont également été identifiés. Ces 56 espèces sont réparties au sein de 19 familles dont celle des Lecanidae est la plus représentée avec 15 espèces (Tableau 3); elle est suivie de la famille des Brachionidae avec 06 espèces. Dans la station $\mathrm{K} 1,30$ espèces zooplanctoniques comprenant 21 de rotifères, 06 de cladocères et 3 de copépodes ont été identifiées durant l'étude. Parmi ces espèces, 04 sont constantes (Rotaria rotaria, Epiphanes macrourus, Lecane bulla et Lepadella patella), 7 sont accessoires et 19 accidentelles. La station K2 est représentée par 33 espèces dont 24 de rotifères, 05 de cladocères, 03 de copépodes et 01 de gastrotriche (Tableau 3). De ces 33 espèces, 08 sont constantes (Rotaria rotaria, Lecane bulla, L. curvicornis, Cephalodella gibba, Mytilina bisulcata, Platyias quadricornis, Tropocyclops confinis, Moina micrura), 06 sont accessoires et 19 sont accidentelles. A la station K3, 52 espèces zooplanctoniques constituées de 36 rotifères, 12 cladocères, 03 copépodes et 01 gastrotriche ont été recensées (Tableau 3). Parmi celles-ci, 22 sont constantes, 12 accessoires et 18 accidentelles. Ainsi, Rotaria rotaria et Lecane bulla sont les seules espèces constantes qui sont apparues à la fois dans les trois stations du Kondi.

Tableau 3 : Abondance moyenne (ind/L) des différentes espèces zooplanctoniques identifiées dans le Kondi avec leur occurrence entre parenthèse

\begin{tabular}{lllll}
\hline Familles & Espèces & \multicolumn{1}{c}{ K1 } & \multicolumn{1}{c}{ K2 } & \multicolumn{1}{c}{ K3 } \\
\hline ROTIFERES & Rotaria macrura (Ehrenberg, 1832) & - & - & $3,21(5)$ \\
Philodinidae & Rotaria neptunia (Ehrenberg, 1832) & $0,21(3)$ & $0,43(6)$ & $26(13)$ \\
& Rotaria rotaria (Pallas, 1766) & $3,43(13)$ & $7,21(13)$ & $131,93(13)$ \\
Epiphanidae & Epiphanes macrourus (Barrois et Daday, 1894) & $0,64(8)$ & $0,43(6)$ & $2,78(8)$ \\
Lecanidae & Lecane bulla (Gosse, 1886) & $1,86(13)$ & $1,28(13)$ & $19,71(14)$ \\
& Lecane candida (Harring \& Myers, 1926) & - & $0,14(2)$ & $0,28(2)$ \\
& Lecane closterocerca (Schmarda, 1859) & $0,28(4)$ & - & $2(9)$ \\
& Lecane cornuta* (O. F. Müller, 1786) & $0,07(1)$ & - & $3,07(2)$ \\
& Lecane curvicornis (Murray, 1930) & $0,14(2)$ & $0,57(7)$ & $2,86(10)$ \\
& Lecane decipiens* (Murray, 1913) & $0,07(1)$ & - & - \\
& Lecane ludwigi* (Eckstein, 1883) & - & - & $1,36(1)$ \\
& Lecane luna (O. F. Müller, 1776) & $0,28(4)$ & - & $2,5(6)$ \\
& Lecane lunaris (Ehrenberg, 1832) & $0,5(6)$ & $0,21(3)$ & $5,21(11)$ \\
& Lecane obtusa (Murray, 1930) & $0,07(1)$ & - & - \\
& Lecane papuana (Murray, 1930) & $0,14(2)$ & $0,43(4)$ & $6,57(8)$ \\
& Lecane pygmea* (Daday, 1897) & $0,14(2)$ & - & - \\
& Lecane quadridentata* (Ehrenberg, 1830) & - & - & $0,36(2)$ \\
& Lecane subtilis (Harring, 1913) & - & - & $0,07(1)$ \\
& Lecane unguilata* (Gosse, 1887) & - & $0,07(1)$ & - \\
& Colurella obtusa (Gosse, 1886) & $0,07(1)$ & $0,86(5)$ & $1,93(7)$ \\
& Colurella uncinata (O. F. Müller, 1776) & - & - & $0,07(1)$ \\
& Lepadella ovalis ( O. F. Müller, 1786) & - & - & $1(6)$ \\
& Lepadella patella (O. F. Müller, 1776) & $0,57(8)$ & $0,21(3)$ & $3,07(13)$ \\
& Euchlanis dilatata (Ehrenberg, 1832) & $0,43(3)$ & $0,21(3)$ & $3,21(12)$ \\
& Dipleuchlanis propatula (Gosse, 1886) & - & $0,21(2)$ & $1,36(10)$ \\
& & & &
\end{tabular}




\begin{tabular}{|c|c|c|c|c|}
\hline $\begin{array}{l}\text { Onana et al. } \\
\text { avec les facte }\end{array}$ & $\begin{array}{l}\text { Appl. Biosci. 2014. Distribution spa } \\
\text { abiotiques dans un hydrosystème urbaiı }\end{array}$ & $\begin{array}{l}\text { nporel } \\
\text { uissea }\end{array}$ & eoplar & en relation \\
\hline Mytilinidae & Mytilina bisulcata (Lucks, 1912) & $0,43(6)$ & $1(11)$ & $2,0710)$ \\
\hline & Mytilina mucronata (O. F. Müller, 1773) & $0,07(1)$ & $0,07(1)$ & $0,21(2)$ \\
\hline & Mytilina ventralis (Ehrenberg, 1832) & - & $0,07(1)$ & $5,14(8)$ \\
\hline & Mytilina trigona (Gosse, 1851) & - & $0,07(1)$ & $0,28(3)$ \\
\hline Brachionidae & Anuraeopsis fissa (Gosse, 1851) & $0,07(1)$ & - & $1,86(4)$ \\
\hline & Plationus patulus (O. F. Müller, 1786) & $0,07(1)$ & $0,07(1)$ & $3,71(5)$ \\
\hline & Plationus patulus patulus* (O. F. Müller, 1786) & - & - & $0,14(1)$ \\
\hline & Platyias leloupi (Gillard, 1957) & - & $0,07(1)$ & $0,93(5)$ \\
\hline & Platyias quadricornis (Ehrenberg, 1832) & - & $0,64(8)$ & $18,43(13)$ \\
\hline & Brachionus quadridentatus (Hermann, 1783) & - & $0,07(1)$ & $0,21(2)$ \\
\hline Scaridiidae & Scaridium grandis* (Sergers, 1995) & - & $0,07(1)$ & $14,64(10)$ \\
\hline Synchaetidae & Polyarthra vulgaris (Carlin, 1943) & - & - & $0,21(2)$ \\
\hline Testudinellidae & Testudinella patina (Hermann, 1783) & - & $0,07(1)$ & $0,71(1)$ \\
\hline Trichocercidae & Trichocerca elongata (Gosse, 1886) & - & - & $0,43(2)$ \\
\hline Notommatidae & Cephalodella gibba (Ehrenberg, 1838) & $0,14(2)$ & $0,57(7)$ & $2,43(6)$ \\
\hline COPEPODES & & & & \\
\hline Cyclopidae & Tropocyclops confinis (Kiefer, 1930) & $0,64(6)$ & $0,57(7)$ & $6,92(14)$ \\
\hline & Afrocyclops gibsoni (Brady, 1904) & $0,43(4)$ & $0,36(5)$ & $0,14(1)$ \\
\hline & Mesocyclops sp & $0,14(2)$ & $0,14(2)$ & $1,5(8)$ \\
\hline & Nauplii & $1,14(12)$ & $0,86(10)$ & $23,64(14)$ \\
\hline & Copépodites & $1,07(10)$ & $0,5(6)$ & $9,43(14)$ \\
\hline CLADOCERES & & & & \\
\hline Moinidae & Moina micrura (Kurz, 1874) & $1,36(4)$ & $1,07(9)$ & $4,64(11)$ \\
\hline Chydoridae & Alona guttata (Sars, 1962) & $0,14(2)$ & $0,93(6)$ & $0,21(2)$ \\
\hline & Alona costata (Sars, 1862) & $0,07(1)$ & $0,07(1)$ & $1,86(4)$ \\
\hline & Kurzia longirostris (Daday, 1898) & - & $0,07(1)$ & $16,57(10)$ \\
\hline & Dunhevedia serrata* (Daday, 1898) & $0,07(1)$ & - & 0,86 \\
\hline & Leydigia ciliata* (Gauthier, 1939) & - & - & $0,14(1)$ \\
\hline Macrothricidae & Guernella raphaelis (Richard, 1892) & - & - & $0,07(1)$ \\
\hline & Grimaldina brazzal* (Richard, 1892) & - & - & $6,64(9)$ \\
\hline & Lathonura rectirostris* (O. F. Müller, 1776) & - & - & $0,43(1)$ \\
\hline & Macrorhrix laticornis (June, 1820) & - & - & $2,43(6)$ \\
\hline Daphniidae & Ceriodaphnia cornuta (Sars, 1886) & $0,07(1)$ & - & $1,57(6)$ \\
\hline Sididae & Diaphanosoma volzi (Stingelin, 1905) & $0,21(3)$ & $0,93(3)$ & $41,64(13)$ \\
\hline GASTROTRICl & & & & \\
\hline Chaetonidae & Chaetonotus sp & - & $0,07(1)$ & $0,57(4)$ \\
\hline
\end{tabular}

${ }^{*}$ : Espèces citées pour la première fois au Cameroun)

Variation spatiale et saisonnière de l'abondance du zooplancton: Des 04 groupes zooplanctoniques récoltés dans le Kondi, les rotifères avec 75,90\% des individus constituent le groupe le plus abondant (Figure 1). Ils sont suivis des cladocères, des copépodes et enfin des gastrotriches (Figure 1). Cette faune est dominée par l'espèce Rotaria rotaria qui représente à elle seule 36,73 $\%$ de la totalité du zooplancton récolté dans le Kondi. Cette espèce est 3,33 fois plus abondante que le cladocère Diaphanosoma volzi $(11,02 \%)$ qui est la deuxième espèce la plus abondante. D'autres espèces se distinguent également par leur abondance il s'agit des 


\section{Onana et al. J. Appl. Biosci. 2014. Distribution spatio-temporelle du zooplancton en relation avec les facteurs abiotiques dans un hydrosystème urbain : le ruisseau Kondi, Cameroun.}

rotifères Rotaria neptunia $(6,86 \%)$, Lecane bulla $(5,89$ $\%)$, Platyias quadricornis (4,92\%), Scaridium grandis $(3,79 \%)$, du cladocère Kurzia longirostris (4,29\%) et du copépode Tropocyclops confinis (2,09\%). Les 48 autres espèces représentent seulement $24,41 \%$ de la faune zooplanctonique. Par ailleurs, l'espèce Rotaria rotaria est prépondérante à toutes les stations avec respectivement $26,78 \%, 37,47 \%$ et $37,04 \%$ des effectifs des stations $\mathrm{K} 1, \mathrm{~K} 2$, et K3. A l'échelle spatiale, avec des valeurs moyennes de 12,81 ind/L, 19,17 ind/L et 356,14 ind/L enregistrées respectivement au niveau des stations $\mathrm{K} 1$, $\mathrm{K} 2$ et K3, les densités zooplanctoniques augmentent de l'amont vers l'aval. Le test de Kruskal-Wallis réalisé à partir des abondances des espèces montre une différence significative d'une part entre les stations K1 et $\mathrm{K} 3$ et d'autre part entre les stations K2 et K3 au seuil de sécurité $5 \%$. Sur le plan temporel, bien que les abondances des espèces varient d'une saison à l'autre, le test de Kruskal -Wallis réalisé à partir des abondances des espèces zooplanctoniques au niveau de chaque station n'a montré aucune différence significative entre la saison sèche et la saison des pluies au même seuil de sécurité.

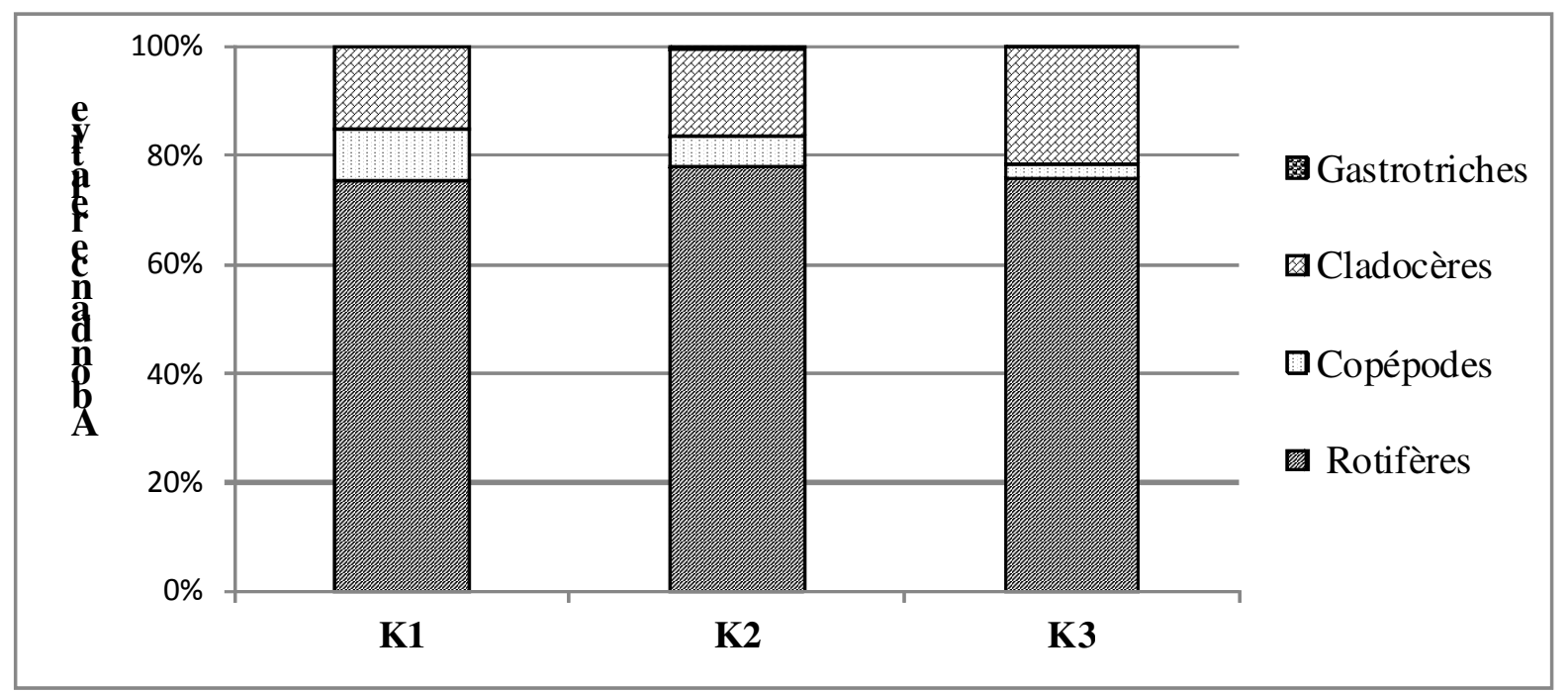

Figure 1 : Contribution des rotifères, des copépodes, des cladocères et des gastrotriches à l'abondance du zooplancton au niveau de chaque station.

Indices de diversité de Shannon et Weaver, indice de Piélou et corrélations :L'indice de diversité de Shannon et Weaver a varié entre 1,58 bit/ind (K2, mai 2012) et 4,13 bit/ind (K3, juillet 2011) (Figure 2) avec des moyennes de $2,64 \pm 0,48,2,63 \pm 0,74$ et $3,35 \pm 0,54$ bits/ind obtenues respectivement dans les stations K1, K2 et K3. L'équitabilité $\mathrm{J}$ pour sa part à varié entre 0,48 (K3, avril 2012) et 1 (K1, octobre 2011 et février 2012) (Figure 2). Cet indice révèle que la station $\mathrm{K} 1$ avec une moyenne de $0,91 \pm 0,1$ renferme une communauté avec des individus qui sont le mieux répartis au sein des différentes espèces. L'application du coefficient de corrélation de rang de Spearmann entre variables Physico-chimiques et biologiques a permis de révéler un certain nombre de liaisons entre les différentes composantes de l'écosystème. En outre, la conductivité électrique est corrélée positivement et significativement $(\alpha=0,05)$ aux espèces suivantes: Rotaria rotaria $(r=0,480)$, Cephaloddella gibba $(r=0,393)$, et Platyias quadricornis $(r=0,425)$. Les teneurs d'azote ammoniacal sont liées aux densités de Rotaria macrura ( $a=0,05 ; r=0,349)$, de Plationus patulus ( $\alpha=0,05 ; r=0,405)$ et de Platyias quadricornis $(\alpha=0,05 ; r=0,335)$. Les orthophosphates sont corrélées positivement à la densité de Mytilina bisulcata ( $\alpha=0,05 ; r=0,314)$. 

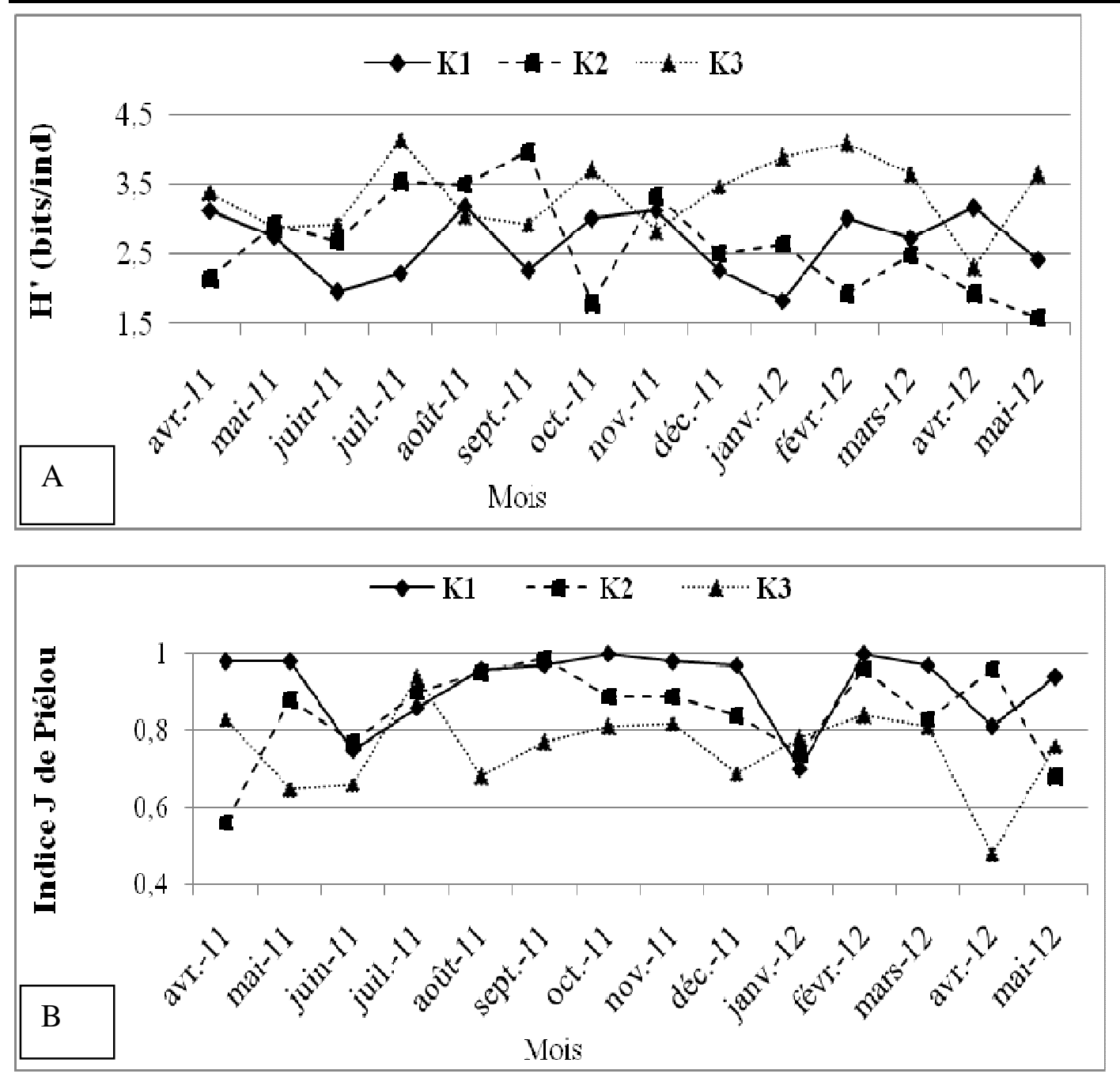

Figure 2 : Variation spatio-temporelle de l'indice de Shannon et Weaver $(A)$ et de l'indice de Piélou (B) dans le Kondi

\section{DISCUSSION}

Caractéristiques physico-chimiques : En se référantà la classification de Goloma et Symoens (1990), les eaux du Kondi sont moyennement (K1) à fortement minéralisées $(\mathrm{K} 2, \mathrm{~K} 3)$. En outre, les valeurs de conductivité électrique enregistrées dans le Kondi sont très élevées par rapport à celles obtenues dans les cours d'eau périurbains et forestiers d'Afrique centrale et de l'ouest par Imoobe et Adeyinka (2009), Gueade et al. (2009), Foto Menbohan et al. (2012) et Foto Menbohan et al. (2013). Ces différences traduisent clairement le fait que l'essentiel des ions enrichissant les cours d'eau urbains est le fait de la minéralisation de la matière organique d'origine anthropique. Contrairement à l'oxygène dissous dont les concentrations baissent à la station K2, les valeurs de la majorité des autres variables (azote ammoniacal, orthophosphates et oxydabilité, conductivité électrique, $\mathrm{pH}, \mathrm{MES}$, turbidité) connaissent une augmentation substantielle à cette station. Cette augmentation résulterait principalement du déversement en amont de la station K2 d'effluents de nature organique provenant principalement de la $\mathrm{SABC}$, de la Cotonnière Industrielle du Cameroun (CICAM) et du marché localisé au lieu-dit PK 8. Priso et al. (2012) incriminent les mêmes sources de pollution alors qu'ils travaillent dans le Kondi. Fagrouch et al. (2011) quant à eux montrent que les valeurs des variables physico-chimiques de l'oued $Z a$ au Maroc subissent une nette augmentation après avoir reçu les effluents de la ville de Taourirt. La légère amélioration observée à la station $\mathrm{K} 3$, située en zone marécageuse, est sans doute le résultat des processus d'autoépuration dont les marécages sont le siège (Fawsi et al., 2001). 


\section{Onana et al. J. Appl. Biosci. 2014. Distribution spatio-temporelle du zooplancton en relation avec les facteurs abiotiques dans un hydrosystème urbain : le ruisseau Kondi, Cameroun.}

Caractéristiques biologiques : La prépondérance des rotifères (40 espèces) sur les microcrustacés (15 espèces) dans les eaux du Kondi est en relation avec les fortes charges en matière organique de ces eaux. Cellesci induisent une multiplication rapide des bactéries (Djuikom, 1998) qui constituent la principale source alimentaire des rotifères (Thouvenot et al., 2000). Cette prépondérance qualitative et quantitative des rotifères a déjà été mise en évidence dans d'autres écosystèmes lotiques tropicaux (Jeje \& Fernando, 1986 ; Egborge \& Tawari, 1987 ; Egborge \& Chigbu, 1988 ; Jeje, 1989 ; Onwudinjo \& Egborge, 1994 ; Fafioye \& Omoyinmi, 2006 ; Foto Menbohan et al., 2006 ; Arimoro \& Oganah, 2010). Les résultats obtenus indiquent une grande variabilité spatiale de la richesse et des abondances des espèces zooplanctoniques qui peuplent les différentes stations d'étude. Aussi, la richesse spécifique élevée et les fortes abondances observées à la station $\mathrm{K} 3$ par rapport aux stations $\mathrm{K} 1$ et $\mathrm{K} 2$ montre que ce tronçon du Kondi offre de meilleures conditions pour le développement du peuplement zooplanctonique. En effet, dans cette station située en zone marécageuse, la grande diversité des habitats, les fortes teneurs en nutriments, l'abondance des ressources alimentaires animales et végétales vivantes et la diminution de la vitesse du courant de l'eau sont favorables au développement et à la prolifération du zooplancton. Ces observations sont en accord avec celles de Vannote et al. (1980), Lecerf (2005) qui ont montré l'efficacité du système fluvial à produire de la matière vivante dans les zones avals des cours d'eau suite à la formation des zones de rétention de la matière organique (bras mort, faciès lentiques). Ces maxima dans les densités zooplanctoniques de la station K3 seraient également une conséquence de la dérive passive des organismes vers les points situés en aval des cours d'eau (Bournaud \& Thibault, 1973). La variabilité saisonnière du zooplancton dans le Kondi n'a pas été très marquée. Cependant, les fortes densités de rotifères sont observées en saison sèche (décembre, janvier et février) par rapport aux périodes de crue (mars à novembre). Cette situation se justifieraient par le fait que pendant la saison sèche, les eaux sont relativement calmes et présentent de nombreux faciès favorables à la prolifération des rotifères; ce qui n'est pas le cas en période de crue où les forts courants et débits sont défavorables à l'installation des organismes planctoniques. Un résultat semblable a été obtenu par Imoobe et Adeyinka (2009) au Nigeria et par Kuczynski (1996) en Argentine. Lair et al. (1998) pour leur part soulignent à ce sujet que les rotifères répondent très bien aux processus d'eutrophisation qui se manifestent dans les rivières en période d'étiage. En effet, seul les taxons de ce groupe de zooplancton possèdent des organismes assez tolérants pour résister à un fort enrichissement du milieu en matière organique et à un appauvrissement en oxygène dissous caractéristiques de l'eutrophisation (Zébazé Togouet, 2008). Les fortes fluctuations spatio-temporelles de l'indice de Shannon et Weaver et de l'équitabilité de Piélou pendant la période d'étude révèlent une grande instabilité dans la structure de la communauté zooplanctonique du Kondi. Ceci montre la variabilité des conditions du milieu qui ont prévalu tout au long de l'étude et du cours d'eau. En outre, les faibles indices d'équitabilité enregistrés au cours de l'étude à la station K3 seraient dus aux fortes abondances relatives des espèces Rotaria rotaria et Diaphanosoma volzi, qui comptent près de $50 \%$ des individus de cette station. En effet, d'après Barbault (1995), MDDEFP (2013) l'indice de Piélou est faible lorsqu'un ou quelques taxons ont une abondance relative très élevée. Parmi les 40 espèces de rotifères identifiés, Rotaria rotaria est dominante à toutes les stations. Cette espèce constituant $36,73 \%$ de la totalité du zooplancton du Kondi et régulièrement constante dans ce cours d'eau, a déjà été abondamment retrouvée dans les cours d'eau eutrophes et hypereutrophes du Cameroun (Foto Menbohan et al., 2006 ; Foto Menbohan, 2012). Cette espèce typique des eaux eutrophes (Zébazé Togouet, 2000) serait donc caractéristique du Kondi. En effet, les eaux du Kondi se distinguent par des fortes quantités de matière organique constituant ainsi des milieux de vie favorables au développement du rotifère Rotaria rotaria qui est reconnue comme indicateur des milieux eutrophes et hypereutrophes (Sladecek, 1983 ; Nogrady et al., 1993 ; Shiel, 1995 ; Zébazé Togouet, 2000 ; Foto Menbohan et al. 2006). Mytilina bisulcata et Cephalodella gibba se font aussi remarquer par leurs abondances élevées. La présence de ces espèces dans un milieu serait révélatrice de leur forte charge en matière organique. Ces résultats sont corroborés par la corrélation positive et significative obtenue entre Mytilina bisulcata et les orthophosphates et entre Cephalodella gibba et la conductivité électrique. Sladecek (1983), a répertorié ces espèces comme indicatrices des eaux mésotrophes à eutrophes. Le peuplement zooplanctonique du Kondi est également caractérisé par l'espèce Lecane bulla qui, bien que numériquement inférieure à Rotaria rotaria, apparait avec de très fortes occurrences $(\mathrm{K} 1=92,86 \%, \mathrm{~K} 2=92,86 \%, \mathrm{~K} 3=100 \%)$. Cette espèce fréquemment rencontrée dans les eaux tropicales eutrophes (Zébazé Togouet, 2000 \& 2008) et à 


\section{Onana et al. J. Appl. Biosci. 2014. Distribution spatio-temporelle du zooplancton en relation avec les facteurs abiotiques dans un hydrosystème urbain : le ruisseau Kondi, Cameroun.}

très faible courant est généralement périphytique (Segers, 1996). Sa prépondérance dans les eaux du Kondi s'expliquerait par le fait que nos échantillonnages ont été effectués au niveau des faciès lentiques des différentes stations, c'est-à-dire où le courant de l'eau est inférieure à $30 \mathrm{~cm}$ par seconde (Malavoi \& Souchon, 2002) et où les herbiers constituent les principaux microhabitats. Hormis les rotifères Rotaria rotaria, Lecane bulla, Cephalodella gibba et Mytilina bisulcata, le cladocère Diaphanosoma volzi se distingue par de fortes densités enregistrées à la station K3. Selon Korovchinsky (1995), cette espèce prolifère abondamment dans les biotopes aquatiques riches en matière organique, à faible profondeur des eaux et où l'on observe une importante végétation (herbier), comme il en est à la station K3.

\section{CONCLUSION}

Les analyses physico-chimiques effectuées sur le Kondi ont révélé que ses eaux présentent une pollution organique forte due aux rejets d'eau usée domestique et industrielle provenant des habitations et des industries localisés dans son bassin versant. La composition taxonomique du zooplancton du Kondi montre que ce sont essentiellement les espèces indicatrices des milieux
D'une manière générale, les grandes quantités de matière organique mise en évidence par les faibles valeurs de I'IPO pourraient expliquer les fortes proportions d'espèces tolérantes à la pollution organique qui ont été dénombrées dans le Kondi au cours de cette étude. II s'agit notamment de Rotaria rotaria, Rotaria neptunia, Lecane bulla, Scaridium grandis, Platyias quadricornis, Kurzia longirostris, Diaphanosoma volzi et Tropocyclops confinis qui constituent $75,59 \%$ du zooplancton. Des résultats similaires ont été obtenus par Zébazé Togouet, (2000 \& 2008) et Foto Menbohan (2012) qui ont noté des fortes abondances de ces espèces dans les hydrosystèmes eutrophes et hypereutrophes du Cameroun.

eutrophes et hypereutrophes qui prolifèrent abondamment dans ce cours d'eau. Cependant, il apparait qu'en plus de l'importante charge en matière organique, la vitesse du courant et la présence de macrophytes sont responsables de la structure des peuplements zooplanctoniques dans ce cours d'eau.

REMERCIEMENTS : Les auteurs remercient Messieurs NNA Samuel et MVONDO Narcisse pour leurs participations aux différentes campagnes d'échantillonnage.

\section{REFEENCES BIBLIOGRAPHIQUES}

American Public Health Association (APHA), 1998. Standard method for examination of water and wastewater. American Public Health Association, 20th edition, Washington, DC, 1150 p.

Amis Ma, Rouget M, Balmford A, Thuiller W, Kleynhans Cj, Day J, Nel J, 2007. Predicting freshwater habitat integrity using land-use surrogates. Water SA, 33: 215- 222.

Arimoro Fo. et Oganah Ao, 2010. Zooplankton Community Responses in a Perturbed Tropical Stream in the Niger Delta, Nigeria. The Open Environmental \& Biological Monitoring Journal, 3, 1-11.

Barbault R, 1995. Ecologie générale: structure et fonctionnement de la biosphère. 5e édition, Dunod, Paris. Pp. 202-205.

Bournaud M. et Thibault M, 1973. La dérive des organismes dans les eaux courantes. Anna. Hydrobiol., 4(1) $11-49$.

BUCREP, 2010. 3ème recensement général de la population et de l'habitat: la population $d u$
Cameroun en 2010. Bureau Central des Recensements et des Etudes de Population du Cameroun, $10 \mathrm{p}$.

Costanza R, D'arge R, Groot R, Grasso M, Hannon B, Limburg K, Naeem S, O'neil Rv, Paruelo J, Raskin Rg, Sutton P, Van Den Belt M, 1997. The value of the world's ecosystem services and natural capital. Nature, 387: 253-260.

Dajoz R, 1982. Précis d'écologie. Bordas, Paris, 505 p.

Djuikom E, 1998. Qualité bactériologique et physicochimique des cours d'eau du réseau $d u$ Mfoundi à Yaoundé. Thèse de Doctorat, Univ. Yaoundé I, Cameroun, $165 \mathrm{p}$.

Durand Jr. et Levêque C, 1980. Flore et Faune aquatique de l'Afrique Sahelo- Soudanienne, ORSTOM, Documentation technique $\mathrm{n}^{\circ} 44$, Tome 1 , Paris, $389 \mathrm{p}$.

Egborge Abm. et Chigbu P, 1988. The Rotifers of Ikpoba River, Bendel State. The Niger. Fld. 53, 117 132.

Egborge Abm. et Tawari PLC, 1987. The rotifers of Warri River, Nigeria. J Plankton Res. 9: 1-13. 


\section{Onana et al. J. Appl. Biosci. 2014. Distribution spatio-temporelle du zooplancton en relation avec les facteurs abiotiques dans un hydrosystème urbain : le ruisseau Kondi, Cameroun.}

Fafioye Oo. et Omoyinmi GAK, 2006. The rotifers of Omi River, Ago-iwoye, Nigeria. Afr. J. Agricult. Res., 1(5): 18 - 188.

Fagrouch A, Berrahou A, El Halouani H, 2011. Impact d'un effluent urbain de la ville de Taourirt sur la structure des communautés de macroinvertébrés de l'oued Za (Maroc oriental). Revue des Sciences de l'Eau. Vol. $24 n^{\circ} 2$. P. 87-101.

Fawzi B, Chlaida M, Oubraim, Loudiki M, Sabour B, Bouzidi A, 2001. Application de certains indices diatomiques à un cours d'eau marocain : Oued Hasser. Revue des Science de l'Eau., 14: 7389.

Fernando Ch, 2002. A guide to tropical freshwater zooplankton: Identification, Ecology and impact on fisheries. Backhuys Publishers, Leiden, 290 p.

Foto Menbohan S, Njine T, Zebaze Togouet Sh, Kemka N, Nola M, Monkiedje A, Boutin C, 2006. Distribution spatiale du zooplancton dans un réseau hydrographique perturbé en milieu urbain tropical (Cameroun). Bull. Soc. Hist. Nat., $142: 53-62$.

Foto Menbohan S, Zebaze Togouet SH, Nyamsi Tchatcho NI, Njine T, 2011a. Evolution spatiale de la diversité des peuplements de macroinvertébrés benthiques dans un cours d'eau anthropisé en milieu tropical (Cameroun). Eur. J. of Sci. Res., 55 (2): 291-300.

Foto Menbohan S, Zebaze Togouet SH, Ajeagah Gideon A, Nzieleu Tchapgnouo GJ, Njine T, 2011b. Ciliates community distribution and saprobic evaluation of an urban hydrosystem: The Biyeme stream Cameroon (Central Africa). J. Appli. Biosci., 48: 3306-3315.

Foto Menbohan S, Koji E, Ajeagah Gideon A, Bilong Nilong CF, Njiné T, 2012. Impact of dam construction on the diversity of benthic macroinvertebrates community in a periurban stream in Cameroon. International Journal of Biosciences. 11: $137-145$.

Foto Menbohan S, 2012. Recherches écologiques sur le réseau hydrographique du Mfoundi (Yaoundé) : Essai de biotypologie. Thèse de Doctorat d'Etat en Biologie des Organismes Animaux, Université de Yaoundé I. Cameroun, $175 p+$ annexes.

Foto Menbohan S, Tchakonte S, Ajeagah G, Zebaze Togouet SH, Bilong Bilong Cf, Njine T, 2013. Water quality assessment using benthic macroinvertebrates in a periurban stream (Cameroon). International Journal of Biotechnology 2(5), 91-104.

Goloma S. et Symoens JJ, 1990. Caractéristiques physiques et chimiques de quelques cours d'eau de Kisangani (Zaïre). Bull. Séanc. Acad. Roy., Sc. Outre-Mer, 35 : 145-157.

Gueade GA, Ouattara A, Edia OE, Goureng G, 2009. Influence de la géologie du milieu sur les caractéristiques physico-chimiques de quelques petits cours d'eau naturels du sud de la Côte d'Ivoire (Afrique de l'ouest). European Journal of Scientific Research 1450-216X (34) : 384-394.

Imoobe Tot. et Adeyinka Ml, 2009. Zooplankton based assessment of the trophic state of a tropical forest river in Nigeria. Arch. Biol. Sci., 61 (4) : 733-740.

Jeje CY, 1989. The cladoceran fauna of Nigeria: A checklist, review of literature and distribution. Rev. Hydrobiology trop. 22 (1), pp 3-11.

Jeje CY, et Fernando $\mathrm{CH}, 1986$. A Practical Guide to the Identification of Nigerian Zooplankton. Kainji Lake Research Institute, Nigeria. 141p.

Korovchinsky NM, (1995). Redescription of Diaphanosoma volzi Stingelin, 1905 (Crustacea: Daphniiformes: Sididae) with remarks on comparative morphology, biology and geographical distribution. Hydrobiologia, Vol 315, Issue 3, pp 189-201.

Koste W, 1978. Rotaria. Die rädertiere mitteleuropus. Borntraegered, Berlin, 2 vols, $673 \mathrm{p}$.

Kramkimel JD, Grifonni U, Kabeya Mukenyi R, 2004. Profil environnemental du Cameroun. AGRIFOR CONSULT. 148p.

Kuczynski D, 1996. Distribution temporal del zooplankton en el rio reconquista (Argentina), con particular referencia a su fauna de rotiferos. Sep. Rev. Fac. Cien. Exac. Qui., $1: 69-93$.

Lair N, Reyes-Marchant P, Jacquet V, 1998. Développement du phytoplancton, des Ciliés et des Rotifères sur deux sites de la Loire moyenne (France), en période d'étiage. Annales de Limnologie, 34 : 35-48.

Lecerf A, 2005. Perturbations anthropiques et fonctionnement écologiques des cours d'eau de tête de bassin: étude du processus de décomposition des litières. Thèse de Doctorat, Université de Toulouse III, France. 176p.

Leclercq L, 2001. Les eaux courantes : caractéristiques et moyens d'étude, dans Les zones humides. Actes des colloques organisés en 1996 par le 
Ministère de la Région Wallonne dans le cadre de l'Année Mondiale des Zones Humides,Jambes, Région Wallonne, DGRNE. pp. 67-82.

Leclercq L. et Maquet B, 1987. Deux nouveaux indices chimique et diatomique de qualité de l'eau courante. Application au Samson et à ses affluents (Bassin de la Meuse Belge). Comparaison avec d'autres indices chimique, biocénotique et diatomique. Inst. Roy. Sci. Nat. Belg. 38:1-112.

Malavoi JR. et Souchon Y, 2002. Description standardisée des principaux faciès d'écoulement observables en rivière : Clé de détermination qualitative et mesures physiques. Bull. Fr. Pêche Piscic. 365/366: 357-372

MDDEFP (Ministère du Développement Durable, de L'environnement, de la Faune et des Parcs), 2013. Guide de surveillance biologique basée sur les macroinvertébrés benthiques d'eau douce du Québec - Cours d'eau peu profonds à substrat grossier, 2013. Direction du suivi de l'état de l'environnement, ISBN 978-2-55069169-3 (PDF), $2^{e}$ édition : $88 \mathrm{p}$.

Moss B, 1998. Ecology of fresh waters: man and medium, past to future. Blackwell Science, Oxford, $557 \mathrm{p}$.

Nogrady T, Wallace Rl, Snell TW, 1993. Rotifera 1: Biology, ecology and systematics. Guide to the identification of the microinvertebrates of the continental water of the world. 4, Dumont H.J. ed., SPB, Academy publisher, The Hague, 142p.

Novotny V, Bartosova A, O'Reilly N, Ehlinger T, 2005. Unlocking the relationship of biotic integrity of impaired waters to anthropogenic stresses. Water Research, 39: 184-198.

Onwudinjo CC. et Egborge ABM, 1994. Rotifers of Benin River, Nigeria. Hydrobiologia; 272: 87-94.

Pourriot R. et Francez AJ, 1986. Rotifères Introduction pratique à la systématique des organismes des eaux continentales françaises 8 , Bulletin Mensuel de la Société Linnéenne de Lyon, 6 : 1-27.

Priso Rj, Oum Go, Din Ndongo, 2012. Utilisation des macrophytes comme descripteurs de la qualité des eaux de la rivière Kondi dans la ville de Douala (Cameroun-Afrique Centrale). J. Appli. Biosci., 53: 3797 - 3811.

Segers H, 1996. The biogeography of littoral Lecane Rotifera. Hydrobiol., 323: 169 - 197.
Shannon Ce. et Weaver W, 1949. The mathematical theory of communication. Urbana University. Press, Illinois, $117 \mathrm{p}$.

Shiel RJ, 1995. A guide to identification of Rotifers, Cladocerans and Copepods from Australian inland water. Édit. CRCFE-MDBC, Albury (Australie). $144 \mathrm{p}$.

Sikati Foko V, 1998. Rejet des stations d'épuration a boues activées à Yaoundé (Cameroun): Physicochimie -microbiologie -essai d'épuration sur station pilote, Thèse de Doctorat de Troisième Cycle, Univ. Yaoundé I, Cameroun, $155 \mathrm{p}$.

Sladecek V, 1983. Rotifers as indicators of water quality. Hydrobiologia, 100 : 169-201.

Suchel J, (1972). Les climats du Cameroun. Thèse de Doctorat $3^{\text {ème }}$ Cycle, Université de Bordeaux III, France, $1186 \mathrm{p}$.

Thouvenot A, Debroas D, Richardot L, Jugnia B, Dévaux $\mathrm{J}, 2000$. A study of changes between years in the structure of plankton community in a newlyflooded reservoir. Arvhiv für Hydrobiologia, $149: 132-152$.

Vannote Rl, Minshall GW, Cummins KW, Sedell JR, Cushing CE, 1980. The River Continuum Concept. Canadian Journal of Fishery Aquatic Science, 37: 130-137.

Zebaze Togouet SH, 2000. Biodiversité et dynamique des populations zooplanctoniques (ciliés, rotifères, cladocères, copépodes) du Lac Municipal de Yaoundé (Cameroun), Thèse de Doctorat de Troisième Cycle, Univ. Yaoundé I, Cameroun, $175 \mathrm{p}$.

Zebaze Togouet Sh, 2008. Eutrophisation et structure de la communauté zooplanctonique du Lac Municipal de Yaoundé. Thèse de Doctorat Ph.D, Faculté des Sciences, Université de Yaoundé I, Cameroun, 200 p. + Annexes. 